\title{
Características Morfológicas y Morfométricas del Músculo Aductor del Hállux y sus Ramos Motores
}

\author{
Morphological and Morphometric Characteristics of the \\ Hallux Adductor Muscle and its Motor Branches
}

Roberto Coronado $^{1,2}$; Aladino Cerda $^{1} \&$ Mariano del Sol $^{3}$

CORONADO, R.; CERDA, A. \& DEL SOL, M. Características morfológicas y morfométricas del músculo aductor del hállux y sus ramos motores. Int. J. Morphol., 37(3):894-899, 2019.

RESUMEN: El hállux se encuentra en aducción en relación al eje del pie y para mantener esta posición requiere de una adecuada alineación ósea, la que está determinada principalmente por la actividad muscular. Una de las estructuras involucradas en esta función es el músculo aductor del hállux, el cual puede producir hállux valgus o hállux rígido cuando ocurre un desbalance en su actividad normal. A pesar de la importancia de este músculo, existen pocos estudios de su complejo neuromuscular. El objetivo de esta investigación fue describir las características morfológicas y morfométricas del músculo aductor del hállux y sus ramos motores en 30 miembros inferiores. Se disecó la planta del pie hasta alcanzar el plano del músculo aductor del hállux y sus ramos motores. La longitud media de la cabeza oblicua del músculo aductor del hállux fue de $78,16 \mathrm{~mm}( \pm 13,35)$ con un ancho máximo promedio de $20,55 \mathrm{~mm}( \pm 2,59)$ y un tendón de $25,87 \mathrm{~mm}( \pm 7,97)$ de longitud. Respecto a las mismas medidas en la cabeza transversa, estas fueron $39,55( \pm 8,26), 15,04$ $( \pm 3,52)$ y $18,51( \pm 10,04)$, respectivamente. La inervación de ambas cabezas del músculo aductor del hállux provenía del ramo profundo del nervio plantar lateral. En la mayoría de las muestras dicho nervio emitió un ramo para la cabeza oblicua y uno para la cabeza transversa. La cabeza oblicua presentaba uno o dos puntos motores, localizados generalmente en su tercio medio. La cabeza transversa presentaba sólo un punto motor localizado frecuentemente en su tercio lateral. El conocimiento de las características morfológicas y morfométricas del músculo aductor del hállux y de sus ramos motores son clínicamente significativos, puesto que permiten realizar una aproximación de la localización del punto motor en los procedimientos electromiográficos.

PALABRAS CLAVE: Músculo aductor del hállux; Nervio plantar lateral; Hállux valgus; Pie.

\section{INTRODUCCIÓN}

Uno de los segmentos anatómicos que presenta mayor interés clínico en el pie es el hállux. Este dedo, en posición normal, se encuentra en aducción en relación al eje del pie. Esta disposición se mantiene principalmente por la actividad del músculo aductor de hállux.

El músculo del aductor del hállux es un músculo corto y plano, intrínseco del pie, forma parte del tercer plano de los músculos plantares (Testut \& Latarjet, 1959; Williams et al., 1995; Faymonville et al., 2012) y está formado por dos cabezas, una oblicua y otra transversa, que poseen distintos orígenes y volúmenes musculares, y que se insertan juntas en el hállux.

La cabeza oblicua es una masa muscular plana que se origina de las bases del II, III, IV hueso metatarsiano y en el tendón del músculo fibular largo (Rouvière \& Delmas, 1999). Además, se puede originar en la cara inferior del hueso cuboides y en el hueso cuneiforme lateral (Testut \& Latarjet). La cabeza transversa se origina de tres o cuatro fascículos en la segunda, tercera y hasta la quinta articulación metatarsofalángica, también de las cápsulas articulares y ligamento metatarsiano profundo correspondiente (Testut \& Latarjet; Williams et al.). Ambas cabezas ocupan un espacio ubicado bajo el primer, segundo, tercer y cuarto hueso metatarsianos y el arco plantar proximal (Williams et al.), insertándose a través de un tendón común en la cápsula articular de la primera articulación metatarsofalángica.

Testut \& Latarjet y Williams et al., señalaron que la cabeza transversa del músculo aductor del hállux envía una proyección al tendón del músculo extensor largo del hállux.

\footnotetext{
${ }^{1}$ Unidad de Anatomía, Departamento de Ciencias Básicas Biomédicas, Facultad de Ciencias de la Salud, Universidad de Talca, Talca, Chile.

${ }^{2}$ Unidad de Anatomía, Departamento de Ciencias Básicas, Facultad de Medicina, Universidad Católica del Maule, Talca, Chile.

${ }^{3}$ Facultad de Medicina, Centro de Excelencia en Estudios Morfológicos y Quirúrgicos (CEMyQ), Universidad de La Frontera, Temuco, Chile.
} 
Owens \& Thordarson (2001), mencionaron que el tendón conjunto de ambas cabezas se une con el tendón de la cabeza profunda del músculo flexor corto del hállux, conteniendo al hueso sesamoideo lateral y llegando juntos a la cápsula articular de la primera articulación metatarsofalángica y a la cara lateral de la base de la falange proximal del hállux.

Por otra parte, para Kura et al. (1997) el vientre muscular de la cabeza oblicua del músculo aductor del hállux es más desarrollado que el de la cabeza transversa. Según Vasconcellos et al. (2011) en un estudio realizado en individuos brasileños, la cabeza oblicua del músculo aductor del hállux mide en promedio $17,50 \mathrm{~mm}( \pm 5,33)$ de ancho en su parte más voluminosa, mientras que la cabeza transversa del músculo aductor del hállux 13,23 mm ( $\pm 2,54)$. Los autores Kura et al. y Vasconcellos et al. señalaron que la longitud media de la cabeza oblicua y transversa del músculo aductor del hállux era de $67,4 \mathrm{~mm}( \pm 4,6) ; 51,45 \mathrm{~mm}( \pm 5,66)$ y $24,8( \pm 4,2) ; 27,04 \mathrm{~mm}( \pm 4,64)$, respectivamente.

La inervación del músculo aductor del hállux está a cargo del ramo profundo del nervio plantar lateral (Testut \& Latarjet y Williams et al.). Esta inervación puede ser a través de un ramo común para ambas cabezas (Akita et al., 1999) o de un ramo individual para cada una de las cabezas (del Sol et al., 1996; Arakawa et al., 2005). De acuerdo con Arakawa et al. (2005), cada cabeza del músculo aductor del hállux recibe un ramo y tiene un punto motor. del Sol et al. reportaron que el ramo para la cabeza oblicua proporciona dos puntos motores (medial y lateral) y el ramo para la cabeza transversa sólo un punto motor.

La acción del músculo aductor del hállux es aducir el hállux y mantener la concavidad inferior de los arcos longitudinal medial, trasversal proximal y distal del tarso (Hakim-Zargar et al., 2010; Groarke et al., 2012). El desbalance en la actividad muscular normal del músculo aductor del hállux puede producir alteraciones como el hállux rígido o hallux valgus (Incel et al., 2002; Arinci Incel et al., 2003; Lowery \& Wukich, 2009; Hakim-Zargar et al.). Estas patologías involucran una alteración en la alineación ósea del hállux, aumentado la carga sobre los elementos articulares (Riegger, 1988), potenciando o disminuyendo la actividad muscular de aquellos músculos que se insertan en el hállux, pudiendo incluso generar compresión nerviosa o vascular (Frink et al., 2010) lo que puede generar episodios dolorosos en esta región (Alshami et al., 2008).

El estudio del complejo neuromuscular del músculo aductor del hállux y el nervio plantar lateral es relativamente poco conocido, por lo que decidimos estudiar las características morfológicas y morfométricas de este músculo junto a sus ramos motores.

\section{MATERIAL Y MÉTODO}

Realizamos un estudio descriptivo transversal en 30 pies de cadáveres humanos adultos, de diferentes nacionalidades, de ambos sexos, fijados y conservados en formaldehido.

Inicialmente se retiró la piel de cada pie junto con el panículo adiposo plantar, realizando una incisión desde los márgenes de la planta del pie hasta llegar a la base de las falanges proximales. Luego se efectuó una incisión transversal en la aponeurosis plantar, entre el tercio proximal y medio, se retiró la aponeurosis plantar, dejando ver el músculo flexor corto de los dedos, el cual fue reclinado distalmente, visualizándose los vasos y nervios plantares laterales, el músculo cuadrado plantar, el tendón del músculo flexor largo de los dedos y los músculos lumbricales. Finalmente, se seccionó los tendones del músculo flexor largo de los dedos, reclinándose distalmente, para disecar el ramo profundo del nervio plantar lateral y sus ramos destinados al músculo aductor del hállux.

Se observó el origen e inserción de ambas cabezas del músculo aductor del hállux. Todas las mediciones se realizaron con un cáliper digital (Stainless Steel®) con precisión de 0,01 $\mathrm{mm}$.

Se midió la longitud, el ancho del vientre muscular en su parte más voluminosa y la longitud del tendón de ambas cabezas del músculo aductor del hállux. Se registraron las relaciones anatómicas de los ramos musculares, el número de ramos musculares, puntos motores y el sitio de ingreso de ellos, en ambas cabezas del músculo aductor del hállux. Se midió la distancia desde el origen del ramo muscular para ambas cabezas del músculo aductor del hállux a la división del nervio plantar lateral. También se midió la distancia desde el punto motor para ambas cabezas del músculo aductor del hállux a la parte lateral de la base de la falange proximal del hállux, para lo cual estas fueron seccionadas en su origen y reclinadas hacia distal.

\section{RESULTADOS}

\section{Características morfológicas del músculo aductor del hállux}

El músculo aductor del hállux en general se presentó corto y plano y en el total de las muestras estaba constituido por dos cabezas, una oblicua y una transversa. 
La cabeza oblicua se originó en el $100 \%$ de los casos de las siguientes estructuras: 1. Base del II hueso metatarsiano, 2. Base del III hueso metatarsiano, 3. Base del IV hueso metatarsiano y 4. Ligamento plantar largo. En un $76,7 \%$ se originó de la cara inferior del hueso cuboides, anterior al canal del mismo y en un 73,3\% de la vaina del tendón del músculo fibular largo (Fig. 1).

La cabeza transversa se originó en las siguientes estructuras: 1. Cápsula de la articulación metatarsofalángica del $2^{\circ}$ dedo en un $60 \%$ de los casos, 2 . Cápsula de la articulación metatarsofalángica del $3^{\circ}$ dedo en un 73,3\% de los casos, 3 . Cápsula de la articulación metatarsofalángica del $4^{\circ}$ dedo en un 56,7 \% de los casos, 5. Cápsula de la articulación metatarsofalángica del dedo mínimo en un 3,3\% de los casos, además del ligamento transverso profundo (Fig. 1).

La inserción del músculo aductor del hállux, en el $100 \%$ de los casos, se realizó a través de un tendón más o menos cilíndrico, que contenía al hueso sesamoideo lateral. Dicha inserción se realizaba a través de un tendón conjunto de las cabezas oblicua y transversa del músculo aductor del hállux y el tendón de la cabeza profunda del músculo flexor corto del hállux, llegando a la cápsula articular de la articulación metatarsofalángica del hállux y a la parte lateral de la base de la falange proximal del hállux.

\section{Características morfométricas del músculo aductor del hállux}

La cabeza oblicua presentó una longitud promedio de 78,16 mm $( \pm 13,35)$, su vientre muscular presentó un ancho máximo promedio de $20,55 \mathrm{~mm}( \pm 2,59)$ y su tendón de inserción fue de 25,87 $\mathrm{mm}$ de largo $( \pm 7,97)$.

La cabeza transversa presentó una longitud promedio de $39,55 \mathrm{~mm}( \pm 8,26)$, su vientre muscular presentó un ancho máximo promedio de $15,04 \mathrm{~mm}( \pm 3,52)$ y su tendón de inserción fue de $18,51 \mathrm{~mm}$ de largo $( \pm 10,04)$.

\section{Características morfológicas de los ramos motores para las cabezas del músculo aductor del hállux}

En el $100 \%$ de las muestras, la inervación para ambas cabezas del músculo aductor del hállux provenía del ramo profundo del nervio plantar lateral. El o los puntos motores para la cabeza oblicua del músculo aductor del hállux se localizaban en su cara profunda, mientras que el punto motor para la cabeza transversa del músculo aductor del hállux se encontraba en su margen posterior (Fig. 2).

En el 73,3\% de las muestras los ramos para las cabezas del músculo aductor del hállux se originaron a dife- rentes niveles, mientras que en el $26,7 \%$ de los casos se originaron a un mismo nivel.

En el 96,7 \% de los casos el ramo profundo del nervio plantar lateral emitió un ramo para la cabeza oblicua del músculo aductor del hállux, mientras que en el 3,3\% restante emitió dos ramos.

En todas las muestras, la cabeza transversa del músculo aductor del hállux, recibió un ramo desde el ramo profundo del nervio plantar lateral. Dicho ramo largo y delgado se relacionaba en su trayecto con la cara plantar de los músculos interóseos.

El número de puntos motores para la cabeza transversa del músculo aductor del hállux era único en el $100 \%$ de los casos, respecto a la cabeza oblicua ésta recibió un solo ramo en el 73,3 \% y dos ramos en el 26,7 \%) de los casos.

Respecto al sitio de penetración de los ramos motores para el músculo aductor del hállux, cuando la cabeza oblicua presentó un punto motor este ramo penetraba en el tercio proximal en el 33,3\% de las muestras o en el tercio medio en el 63,3\% de los casos; cuando presentaba dos puntos motores, el segundo se encontraba en el tercio proximal del músculo en el $37,5 \%$ de los casos o en el tercio medio en el 62,5\% de los casos. El punto motor de la cabeza transversa en la mayoría de las muestras se encontraba en el tercio lateral de ella $(96,6 \%)$ y solamente en un caso se encontó en el tercio intermedio $(3,4 \%)$.

\section{Características morfométricas de los ramos motores para las cabezas del músculo aductor del hállux}

La distancia promedio desde el origen del ramo muscular para la cabeza oblicua del músculo aductor del hállux a la división del nervio plantar lateral fue de $21,80 \mathrm{~mm}$ $( \pm 6,43)$, mientras que la distancia promedio desde el origen del ramo muscular para la cabeza transversa del músculo aductor del hállux a la división del nervio plantar lateral fue de $24,47 \mathrm{~mm}( \pm 5,56)$.

Cuando había solamente un punto motor para la cabeza oblicua del músculo aductor del hállux, este se encontraba a $48,02 \mathrm{~mm}( \pm 7,64)$ de la parte lateral de la base de la falange proximal del hállux. Cuando había dos puntos motores, el segundo se localiza a 46,49 $\mathrm{mm}( \pm 11,02)$ de la parte lateral de la base de la falange proximal del hállux. El punto motor para la cabeza transversa se encontraba localizado a $33,96 \mathrm{~mm}( \pm 4,12)$ de la parte lateral de la base de la falange proximal del hállux. 


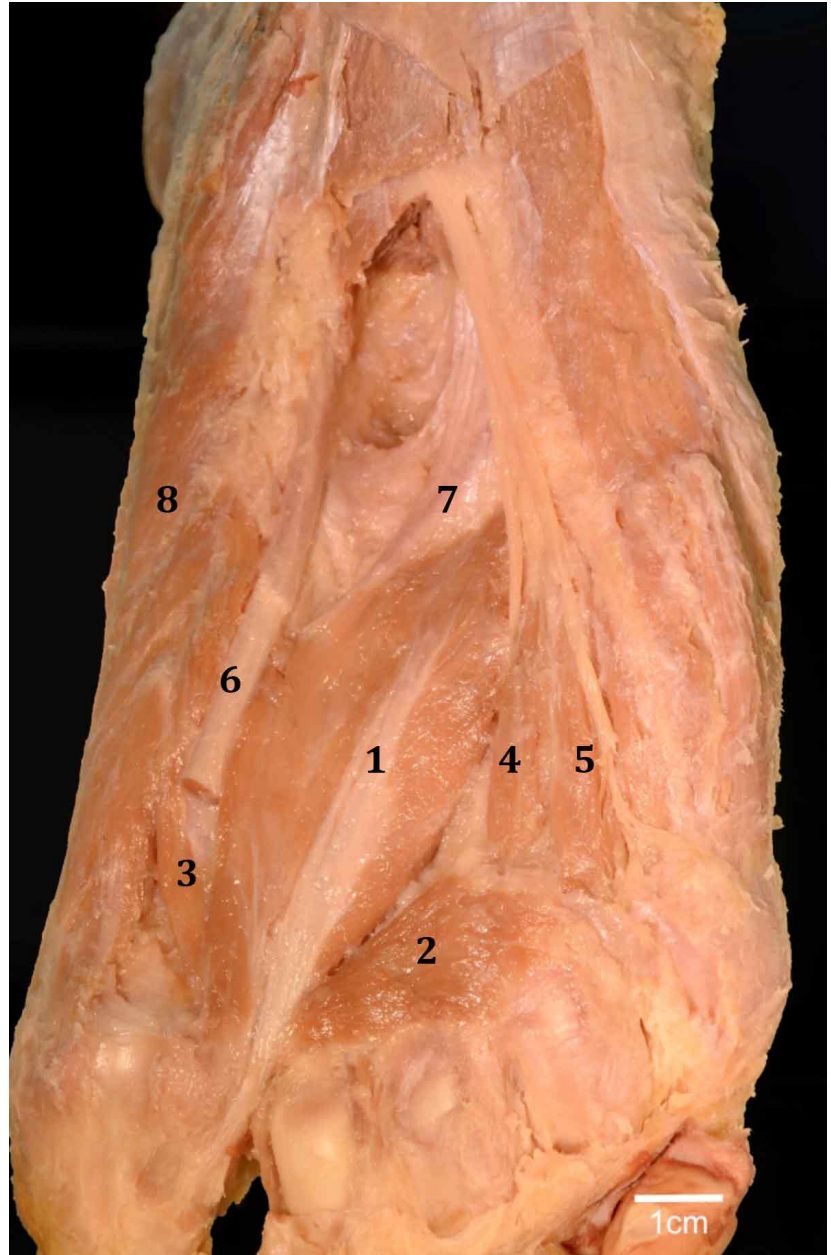

Fig. 1. Músculos plantares. 1. Cabeza oblicua del m. aductor del hállux; 2. Cabeza transversa del m. aductor del hállux; 3. Cabeza profunda del m. flexor corto del hállux; 4. IV m. interóseo dorsal; 5. III m. interóseo plantar; 6. Tendón del m. flexor largo del hállux (seccionado); 7. Tendón del m. fibular largo; 8. M. abductor del hállux.

\section{DISCUSIÓN}

El músculo aductor del hállux, debido a su disposición en la región plantar, es un elemento importante tanto en la alineación de la primera articulación metatarsofalángica como en la mantención de los arcos plantares transversal distal y longitudinal medial (Riegger). Las variaciones en su origen o inserción pueden determinar patologías del hállux como el hállux valgus, hállux rígido, entre otras (Lowery \& Wukich).

Nuestros resultados respecto al origen de la cabeza oblicua del músculo aductor del hállux coinciden con los reportados por Arakawa et al. (2003), sin embargo, esto no ocurre con algunos autores. Williams et al., no hacen men-

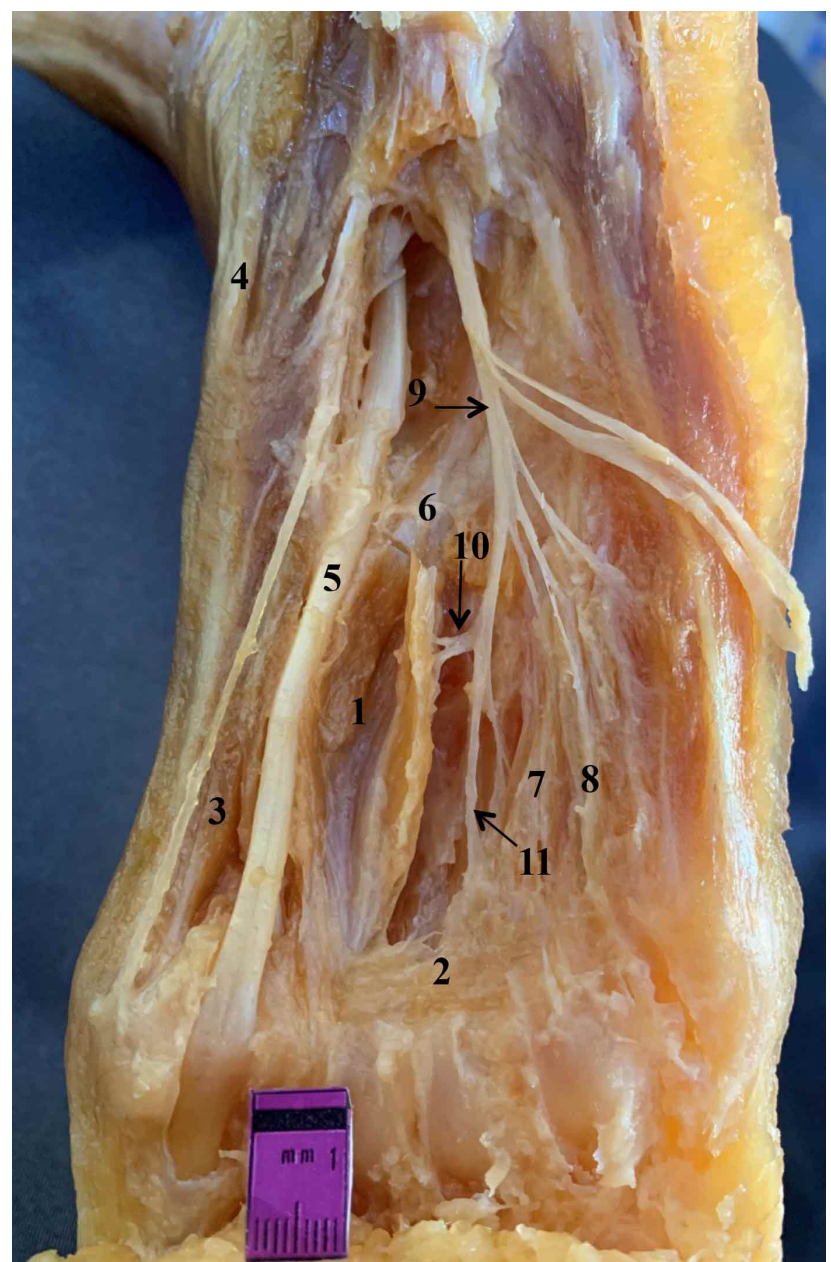

Fig. 2. Músculos plantares y ramo profundo del nervio plantar lateral con la cabeza oblicua del $\mathrm{m}$. aductor del hállux desplazada medialmente. 1. Cabeza oblicua del m. aductor del hállux; 2. Cabeza transversa del $\mathrm{m}$. aductor del hállux; 3. Cabeza superficial del m. flexor corto del hállux; 4. M. abductor del hállux; 5. Tendón del m. flexor largo del hállux; 6. Tendón del m. fibular largo; 7. IV m. interóseo dorsal; 8. III m. interóseo plantar; 9. Ramo profundo del nervio plantar lateral; 10. Ramo motor y puntos motores de la cabeza oblicua del m. aductor del hállux; 11 . Ramo motor y punto motor de la cabeza transversa del $\mathrm{m}$. aductor del hállux.

ción al origen de la cabeza oblicua del músculo aductor del hállux en la cara inferior del hueso cuboides. Moore \& Dalley (2007) y Testut \& Latarjet, no mencionan un origen en el ligamento plantar largo. Vasconcellos et al., no reportaron orígenes de la cabeza oblicua del músculo aductor del hállux en el hueso cuboides ni en la vaina del tendón del músculo fibular largo.

El origen más común de la cabeza transversa del músculo aductor del hállux se realizó en las articulaciones metatarsofalángicas II, III y IV, lo que concuerda con lo 
expresado en los textos de anatomía de Testut \& Latarjet; Williams et al.; Riegger; Rouvière \& Delmas; Moore \& Dalley. Por otra parte, Vasconcellos et al., indicaron que el origen más común de la cabeza transversa del músculo aductor del hállux, se encuentra en la II y III articulaciones metatarsofalángicas y sólo en casos aislados en la $\mathrm{V}$ articulación metatarsofalángica, coincidentes con los resultados de este estudio.

Respecto a la inserción, los resultados son similares a los descritos por Owens \& Thordarson y semejantes a lo señalado por Williams et al. y Moore \& Dalley, quienes, no describieron su inserción en la cápsula articular de la articulación metatarsofalángica del hállux.

El ancho promedio de las cabezas oblicua y transversa del músculo aductor del hállux, es similar a lo informado por Vasconecellos et al. y la longitud media de la cabeza oblicua es semejante a lo reportado por Kura et al. y superior a lo descrito por Vasconcellos et al. Dicha diferencia se puede explicar por el origen étnico distinto de los sujetos estudiados. La longitud media de la cabeza transversa del músculo aductor del hállux es similar a la indicada en los estudios realizados por Kura et al. y Vasconcellos et al.

Coincidimos con los autores del Sol et al. y Arakawa et al. (2003), en que las dimensiones de la cabeza oblicua del músculo aductor del hállux son mayores que las de la cabeza transversa. Kura et al. indicaron que la cabeza oblicua puede ser hasta 9 veces más voluminosa que la transversa. Esta diferencia de dimensiones se debe posiblemente a la postura bípeda que logró el hombre (Gebo et al., 1996; Schmitt et al., 2002). Se ha registrado que en primates las dimensiones de ambas cabezas del músculo aductor del hállux se tienden a igualar, debido a la función de prensión que tienen los pies en estas especies (Suzuki et al., 1982; Inokuchi, 1985).

En el total de las muestras pudimos observar que la inervación del músculo aductor del hállux provenía directamente del ramo profundo del nervio plantar lateral. Con respecto a la inervación de la cabeza oblicua, el o los ramos motores ingresaban al músculo por su cara profunda, no por su cara superficial como señalaron del Sol et al. en sujetos brasileños, a pesar que, como en la mayoría de los casos, la inervación de los músculos profundos se realiza casi siempre por su cara superficial, contrario a lo que ocurre con la inervación de los músculos superficiales. Respecto a la inervación de la cabeza transversa, en ninguna de nuestras muestras encontramos un ramo común que inervara tanto a la cabeza transversa como a los músculos lumbricales II y III y primeros dos músculos interóseos dorsales tal como señalaron Arakawa et al. (2005). El origen común de estos ramos puede estar relacionado con el origen embriológico de dichos músculos. La cabeza oblicua se desarrolla desde el mismo primordio embriológico que el músculo flexor corto del hállux y la cabeza transversa lo hace a través de un origen común con los músculos lumbricales e interóseos (Cihak, 1972).

Coincidimos con del Sol et al. y Arawaka et al. (2005), respecto a que la inervación para cada una de las cabezas es entregada por ramos individuales. Respecto al número de ramos motores, en la mayoría de los casos la cabeza oblicua recibía un ramo motor y tenía un punto motor. En aquellos casos en los que la cabeza oblicua del músculo aductor del hállux tenía dos puntos motores, de acuerdo a su ubicación, estos se podrían describir como medial y lateral tal como lo plantearon del Sol et al. Concordamos con los autores Arawaka et al. (2005) y del Sol et al. respecto a que la cabeza transversa del músculo aductor del hállux tiene solamente un ramo motor y un punto motor.

No encontramos datos en la literatura respecto al sitio de penetración del ramo motor en relación a la longitud de la cabeza muscular. Dicha información es relevante para realizar intervenciones clínicas como la electromiografía de agujas (Lee et al., 2012) o la inyección de toxina botulínica (Minetto \& Botter, 2009) entre otras.

En cuanto a la distancia promedio desde el origen del ramo muscular para cada una de las cabezas, no encontramos datos en la literatura consultada. En 8 de las 30 muestras los ramos musculares para las cabezas oblicua y transversa del músculo aductor del hállux se originaron a un mismo nivel. Tampoco encontramos datos de autores que hayan medido la distancia desde el o los puntos motores de cada una de las cabezas del músculo aductor del hállux a la parte lateral de la base de la falange proximal del hállux, dicha información es relevante a la hora de ubicar el punto motor de cada una de las cabezas al tomar un punto de referencia óseo bastante fácil de palpar.

Debido a la importancia del músculo aductor del hállux tanto en la alineación de la primera articulación metatarsofalángica así como en la mantención de los arcos plantares, el conocimiento de su complejo neuromuscular es clínicamente significativo. Conociendo los sitios de origen e inserción más común de cada una de las cabezas se puede realizar una aproximación de la longitud de cada una de ellas y en base a esta medida, establecer en cual de los tercios se encontraría el punto motor. En base a nuestros resultados podemos indicar que el sitio más común para la localización del o los puntos motores de la cabeza oblicua es su tercio medio, mientras que el sitio más común para la localización del punto motor de la cabeza transversa es su tercio lateral. 
CORONADO, R.; CERDA, A. \& DEL SOL, M. Morphological and morphometric chacarteristics of the hállux adductor muscle and its motor branches. Int. J. Morphol., 37(3):894-899, 2019.

SUMMARY: The hallux is adducted in relation to the axis of the foot and to maintain this position requires adequate bone alignment, which is determined mainly by muscle activity. One of the structures that is involved in this function is the adductor muscle of the hallux, which can produce hallux valgus or rigid hallux when an imbalance occurs in its normal activity. Despite the importance of this muscle, there are few studies of its neuromuscular complex. The objective of this study was to describe the morphological and morphometric characteristics of the adductor muscle of the hallux and its motor branches in 30 lower limbs. The sole of the foot was dissected until it reached the plane of the muscle and its motor branches. The average length of the oblique head of the adductor muscle of the hallux was $78.16 \mathrm{~mm}( \pm 13.35)$, with an average maximum width of $20.55 \mathrm{~mm}( \pm 2.59)$ and a tendon of $25.87 \mathrm{~mm}$ $( \pm 7,97)$ in length. Regarding the same measurements of the transverse head were $39.55( \pm 8.26), 15.04( \pm 3.52)$ and $18.51( \pm$ $10.04)$, respectively. The innervation of both heads came from the deep branch of the lateral plantar nerve. In most of the samples, said nerve emitted a bouquet for the oblique head and one for the transverse head. The oblique head had one or two motor points, generally located in its middle third. The transverse head had only one motor point that was usually in its lateral third. The knowledge of the morphological and morphometric characteristics of the adductor muscle of the hallux and its motor branches are clinically significant, since they allow an approximation of the location of the motor point in electromyographic procedures.

KEY WORDS: Adductor hallucis muscle; Lateral plantar nerve; Hallux valgus; Foot.

\section{REFERENCIAS}

Akita, K.; Sakamoto, H. \& Sato, T. Lateromedial and dorsoplantar borders among supplying areas of the nerves innervating the intrinsic muscles of the foot. Anat. Rec., 255(4):465-70, 1999.

Alshami, A. M.; Souvlis, T. \& Coppieters, M. W. A review of plantar heel pain of neural origin: differential diagnosis and management. Man. Ther., 13(2):103-11, 2008.

Arakawa, T.; Sekiya, S.; Kumaki, K. \& Terashima, T. Ramification pattern of the deep branch of the lateral plantar nerve in the human foot. Ann. Anat., 187(3):287-296, 2005.

Arakawa, T.; Tokita, K.; Miki, A. \& Terashima, T. Anatomical study of human adductor hallucis muscle with respect to its origin and insertion. Ann. Anat., 185(6):585-92, 2003.

Arinci Incel, N.; Genc, H.; Erdem, H. R. \& Yorgancioglu, Z. R. Muscle imbalance in hallux valgus: an electromyographic study. Am. J. Phys. Med. Rehabil., 82(5):345-9, 2003.

Cihak, R. Ontogenesis of the skeleton and intrinsic muscles of the human hand and foot. Ergeb Anat. Entwicklungsgesch., 46(1):5-194, 1972.

Del Sol, M.; Prates, J. C. ; Olave, E.; Mandiola, E. \& Gabrielli, C. Inervación del músculo aductor del hálux. Rev. Chil. Anat., 14(1):73-8, 1996.

Faymonville, C.; Andermahr; Seidel, U.; Muller, L.P.; Skouras, E.; Eysel P. \& Stein,G. Compartment of foot: topographic anatomy. Surg. Radiol. Anat., 34:929-33, 2012.
Frink, M.; Hildebrand, F.; Krettek, C.; Brand, J. \& Hankemeier, S. Compartment syndrome of the lower leg and foot. Clin. Orthop. Relat. Res., 468(4):940-50, 2010.

Gebo, D. L. Climbing, brachiation, and terrestrial quadrupedalism: historical precursors of hominid bipedalism. Am. J. Phys. Anthropol., 101(1):5592, 1996.

Groarke, P.; Galvin, R.; Kelly, J. \& Stephens, M. M. Quality of life in individuals with chronic foot conditions: a cross sectional observational study. Foot (Edinb)., 22(2):66-9, 2012.

Hakim-Zargar, M.; Aronow, M. S.; Gibson, L. \& Obopilwe, E. Implications for the anatomy of the flexor hallucis brevis insertion. Foot Ankle Int. 31(1):65-8, 2010.

Incel, N. A.; Genc, H.; Yorgancioglu, Z. R. \& Erdem, H. R. Relation between hallux valgus deformity and lumbar and lower extremity biomechanics. Kaohsiung J. Med. Sci., 18(7):329-333, 2002.

Inokuchi S. Primate hands and feet: comparative myology. In: Kondo, S. (Ed). Primate Morphophysiology, Locomotor Analyses and Human Bipedalism. Tokyo, University of Tokyo Press, 1985. pp. 153-66,

Kura, H.; Luo, Z. P.; Kitaoka, H. B. \& An, K. N. Quantitative analysis of the intrinsic muscles of the foot. Anat. Rec., 249(1):143-51, 1997.

Lee, J. H.; Han, S. H.; Ye, J. F.; Lee, B. N.; An, X. \& Kwon, S. O. Effective zone of botulinum toxin a injections in hallux claw toe syndrome: an anatomical study. Muscle Nerve., 45(2):217-21, 2012.

Lowery, N. J. \& Wukich, D. K. Adolescent Hallux Valgus: Evaluation and Treatment. Op. Tech. Orthop., 19(1):52-7, 2009.

Minetto, M. A. \& Botter, A. Elicitability of muscle cramps in different leg and foot muscles. Muscle Nerve, 40(4):535-44, 2009.

Moore, K. \& Dalley, A. Anatomía con Orientación Clínica. 5. ed. Buenos Aires, Médica Panamericana, 2007.

Owens, S. \& Thordarson, D. B. The adductor hallucis revisited. Foot Ankle Int., 22(3):186-91, 2001.

Riegger, C. L. Anatomy of the Ankle and foot. Physical Therapy., 68(12):180214, 1988

Rouvière, H. \& Delmas, A. Anatomía Humana. Vol. 2. 10ª ed. Barcelona, Masson, 1999.

Schmitt, D. \& Lemelin, P. Origins of primate locomotion: gait mechanics of the woolly opossum. Am J. Phys. Anthropol., 118(3):231-8, 2002.

Suzuki, M.; Inokuchi, S.; Nakanishi, H.; Ajiri, T.; Kimura, T. \& Matsumoto, Y. Comparative anatomical studies of myofibrous organization of monkey and human hand and foot muscles especially monkey foot muscles. $J$. Showa Med. Assoc., 42:737-46, 1982.

Testut, L. \& Latarjet, A. Tratado de Anatomía Humana. 9ª ed. Barcelona, Salvat, 1959

Vasconcellos, H. A.; Cavalcante, M. L. T. M. H.; Ferreira, I. G.; Frango, A. O. R.; Siqueira, C. O. \& Galvao, J. A. El cambio de la forma del músculo aductor hallux y el hallux valgus. Int. J. Morphol., 29(4):1303-6, 2011.

Williams, P.; Warwick, R.; Dyson, M. \& Bannister, L. Gray Anatomía. Vol. 1. $37^{a}$ ed. São Paulo, Guanabara Koogan, 1995.

Dirección para correspondencia:

Dr. Mariano del Sol Calderón

Facultad de Medicina

Universidad de La Frontera

Temuco

CHILE

Email: mariano.delsol@ufrontera.cl

Recibido : 01-03-2019

Aceptado: 20-04-2019 